\title{
State-Dependent Z-Interference Channel with Correlated States
}

\author{
Yunhao Sun, Yingbin Liang \\ Dept of EECS, Syracuse University \\ Syracuse, NY 13244, USA \\ Email: \{ysun33,yliang06\}@ syr.edu
}

\author{
Ruchen Duan \\ Samsung Semiconductor Inc. \\ San Diego, CA 92121, USA \\ Email: r.duan@samsung.com
}

\author{
Shlomo Shamai (Shitz) \\ Dept of EE, Technion \\ Technion city, Haifa 32000, Israel \\ Email:sshlomo@ee.technion.ac.il
}

\begin{abstract}
This paper investigates the Gaussian statedependent $\mathrm{Z}$-interference channel (Z-IC), in which two receivers are corrupted respectively by two correlated states that are noncausally known to transmitters and unknown to receivers. Three interference regimes are studied, and the capacity region or sum capacity boundary is characterized either fully or partially under various channel parameters. The impact of correlation between the states on state and interference cancellation as well as the achievability of the capacity is demonstrated via numerical analysis.
\end{abstract}

\section{INTRODUCTION}

State-dependent interference channels (ICs) are of great interest in wireless communications, in which receivers are interfered not only by other transmitters' signals but also by independent and identically distributed (i.i.d.) state sequences. The state can capture interference signals that are informed to transmitters, and are hence often assumed to be noncausally known by these transmitters in the model. Such interference cognition can occur in practical wireless networks due to node coordination or backhaul networks.

Both the state-dependent IC and Z-IC have been studied in the literature. The state-dependent IC was studied in [1], [2] with two receivers corrupted by the same state, and in [3] with two receivers corrupted by independent states. In [4], [5], two state-dependent cognitive IC models were studied, where one transmitter knows both messages, and the two receivers are corrupted by two states. More recently, in [6], both the state-dependent regular IC and Z-IC were studied, where the receivers are corrupted by the same but differently scaled state. Furthermore, in [7], [8], a type of the statedependent Z-IC was studied, in which only one receiver is corrupted by the state and the state information is known only to the other transmitter. In [9], a type of the state-dependent Z-IC with two states was studied, where each transmitter knows only the state that corrupts its corresponding receiver. In [10], a state-dependent Z-interference broadcast channel was studied, in which one transmitter has only one message for its corresponding receiver, and the other transmitter has two messages respectively for two receivers. Both receivers are corrupted by the same state, which is known to both transmitters.

In all the previous work of the state-dependent IC and $\mathrm{Z}$-IC, the states at two receivers are either assumed to be independent, or to be the same but differently scaled, with the exception of [9] that allows correlation between states. However, [9] assumes that each transmitter knows only one state at its corresponding receiver, and hence two transmitters cannot cooperate to cancel the states. In this paper, we investigate the state-dependent $\mathrm{Z}$-IC with the two receivers being corrupted respectively by two correlated states and with both transmitters knowing both states in order for them to cooperate. The state sequences are assumed to be known at both transmitters. The main focus of this paper is on the Gaussian state-dependent Z-IC, where the receivers are corrupted by additive interference, state, and noise. The aim is to design encoding and decoding schemes to handle interference as well as to cancel the state at the receivers. In particular, we are interested in answering the following two fundamental questions: (1) whether or under what conditions both states can be simultaneously fully canceled so that the capacity for the Z-IC without state can be achieved; and (2) what is the impact that the correlation between two states make towards state cancellation and capacity achievability.

We summarize our results as follows. Our novelty of designing achievable schemes lies in joint design of the interference cancellation schemes together with the Gel'fandPinsker binning [11] and dirty paper coding [12] for state cancellation in order to characterize the capacity region. More specifically, we study three interference regimes. For the very strong interference regime, we characterize the channel parameters under which the two receivers achieve their corresponding point-to-point channel capacity without state and interference. Thus, the interference as well as states are fully canceled, and the capacity region is characterized as a rectangular region. In particular, we demonstrate the impact of the correlation between the two states in such a regime. Interestingly, we demonstrate that high interference may not always be beneficial for canceling both state and interference, which is in contrast to the IC without state. For the strong regime, we characterize the sum capacity boundary partially under certain channel parameters based on joint design of rate splitting, successive cancellation, as well as dirty paper coding. For the weak interference regime, we characterize the sum capacity, which is achieved by the two transmitters independently designing dirty paper coding and receiver 1 treating interference as noise. The sum capacity is not affected 
by the correlation between states.

\section{Channel Model}

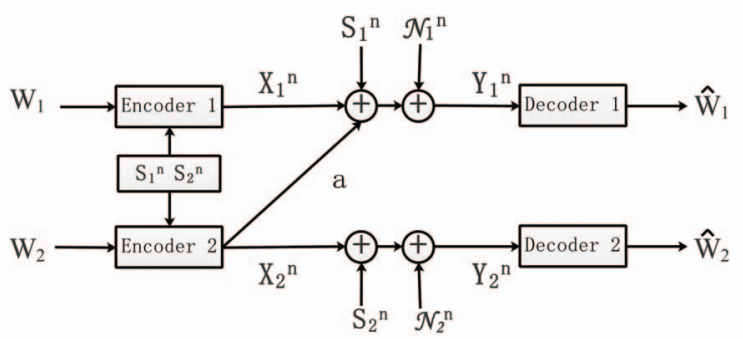

Fig. 1. The state-dependent Z-IC

We consider the state-dependent Z-IC (as shown in Fig. 1), in which transmitters 1 and 2 send two messages $W_{1}$ and $W_{2}$ to two receivers 1 and 2, respectively. Receiver 1's output is interfered by transmitter 2's input as well as a state sequence $S_{1}^{n}$, and receiver 2's output is interfered only by a state sequence $S_{2}^{n}$, which is correlated with $S_{1}^{n}$. The two state sequences $S_{1}^{n}$ and $S_{2}^{n}$ are assumed to be known noncausally at both transmitters. The encoder $k$ at transmitter $k$ maps the message $w_{k} \in \mathcal{W}_{k}=\left\{1, \ldots, 2^{n R_{k}}\right\}$ and the state sequences $s_{1}^{n}$ and $s_{2}^{n}$ to a codeword $x_{k}^{n} \in \mathcal{X}_{k}^{n}$ for $k=1,2$. The two inputs $x_{1}^{n}$ and $x_{2}^{n}$ are transmitted over the memoryless ZIC characterized by $P_{Y_{1} \mid X_{1} X_{2} S_{1}}$ and $P_{Y_{2} \mid X_{2} S_{2}}$. Receiver 1 is required to decode $W_{1}$ and receiver 2 is required to decode $W_{2}$. The average probability of error for a length- $n$ code is defined as

$$
P_{e}^{(n)}=\frac{1}{\left|\mathcal{W}_{1}\right|\left|\mathcal{W}_{2}\right|} \sum_{w_{1}=1}^{\left|\mathcal{W}_{1}\right|} \sum_{w_{2}=1}^{\left|\mathcal{W}_{2}\right|} \operatorname{Pr}\left\{\left(\hat{w}_{1}, \hat{w}_{2}\right) \neq\left(w_{1}, w_{2}\right)\right\} .
$$

A rate pair $\left(R_{1}, R_{2}\right)$ is achievable if there exist a sequence of encoding and decoding schemes such that the average error probability $P_{e}^{(n)} \rightarrow 0$ as $n \rightarrow \infty$. The capacity region is defined to be the closure of the set of all achievable rate pairs.

In this paper, we focus on the Gaussian Z-IC with the outputs at the two receivers for one channel use given by

$$
\begin{aligned}
& Y_{1}=X_{1}+a X_{2}+S_{1}+N_{1} \\
& Y_{2}=X_{2}+S_{2}+N_{2}
\end{aligned}
$$

where $a$ is the channel gain coefficient, and $N_{1}$ and $N_{2}$ are noise variables with Gaussian distributions $N_{1} \sim \mathcal{N}(0,1)$ and $N_{2} \sim \mathcal{N}(0,1)$. The state variables $S_{1}$ and $S_{2}$ are jointly Gaussian with correlation coefficient $\rho$ and marginal distributions $S_{1} \sim \mathcal{N}\left(0, Q_{1}\right)$ and $S_{2} \sim \mathcal{N}\left(0, Q_{2}\right)$. Both the noise variables and the state variables are i.i.d. over channel uses. The channel inputs $X_{1}$ and $X_{2}$ are subject to the average power constraints $P_{1}$ and $P_{2}$.

Our goal is to characterize channel parameters, under which the capacity of the corresponding Z-IC without the presence of the states can be achieved, and thus the capacity region of the Z-IC with the presence of state is also established. In particular, we are interested in understanding the impact of the correlation between the states on the capacity characterization.

\section{VERy Strong InTERFERENCE REGIME}

In this section, we study the state-dependent Z-IC in the very strong regime, in which the channel parameters satisfy $a^{2}>1+P_{1}$. For the corresponding Z-IC without states, the capacity region contains rate pairs $\left(R_{1}, R_{2}\right)$ satisfying

$$
R_{1} \leqslant \frac{1}{2} \log \left(1+P_{1}\right), \quad R_{2} \leqslant \frac{1}{2} \log \left(1+P_{2}\right) .
$$

In this case, the two receivers achieve the point-to-point channel capacity without interference. Furthermore, in [6], an achievable scheme has been established to achieve the same point-to-point channel capacity when the two receivers are corrupted by the same but differently scaled state. Our focus here is on the more general scenario, where the two receivers are corrupted by two correlated states, and our aim is to understand how the correlation affects the design of the scheme.

We first design an achievable scheme to obtain an achievable rate region for the discrete memoryless Z-IC. The two transmitters encode their messages $W_{1}$ and $W_{2}$ into two auxiliary random variables $U$ and $V$, respectively, based on the Gel'fand-Pinsker binning scheme. Since receiver 2 is interference free and is corrupted by $S_{2}$, the auxiliary random variable $V$ is designed with regard to only $S_{2}$. Furthermore, receiver 1 first decodes $V$, then uses it to cancel the interference $X_{2}$ and partial state interference, and finally decodes its own message $W_{1}$ by decoding $U$. Here, since $S_{2}$ is introduced to $Y_{1}$ when canceling $X_{2}$ via $V$, the auxiliary random variable $U$ is designed based on both $S_{1}$ and $S_{2}$ to fully cancel the states. Based on such a scheme, we obtain the following achievable region.

Proposition 1. For the state-dependent Z-IC with the states noncausally known at both transmitters, an achievable region consists of rate pairs $\left(R_{1}, R_{2}\right)$ satisfying:

$$
\begin{aligned}
& R_{1} \leqslant I\left(U ; V Y_{1}\right)-I\left(S_{1}, S_{2} ; U\right) \\
& R_{2} \leqslant \min \left\{I\left(V ; Y_{2}\right), I\left(V ; Y_{1}\right)\right\}-I\left(S_{2} ; V\right)
\end{aligned}
$$

for some distribution $P_{S_{1} S_{2}} P_{U \mid S_{1} S_{2}} P_{X_{1} \mid U S_{1} S_{2}} P_{V \mid S_{2}} P_{X_{2} \mid V S_{2}}$ $P_{Y_{1} \mid S_{1} X_{1} X_{2}} P_{Y_{2} \mid S_{2} X_{2}}$.

The proof of Propostion 1 is omitted due to the page limitations.

Following Proposition 1, we further simplify the achievable region in the following corollary, which is in a useful form for us to characterize the capacity region for the Gaussian Z-IC.

Corollary 1. For the state-dependent Z-IC with the states noncausally known at both transmitters, if the following condition

$$
I\left(V ; Y_{2}\right) \leqslant I\left(V ; Y_{1}\right)
$$

is satisfied, then an achievable region consists of rate pairs $\left(R_{1}, R_{2}\right)$ satisfying:

$$
\begin{aligned}
& R_{1} \leqslant I\left(U ; V Y_{1}\right)-I\left(S_{1}, S_{2} ; U\right) \\
& R_{2} \leqslant I\left(V ; Y_{2}\right)-I\left(S_{2} ; V\right)
\end{aligned}
$$


for some distribution $P_{S_{1} S_{2}} P_{U \mid S_{1} S_{2}} P_{X_{1} \mid U S_{1} S_{2}} P_{V \mid S_{2}} P_{X_{2} \mid V S_{2}}$ $P_{Y_{1} \mid S_{1} X_{1} X_{2}} P_{Y_{2} \mid S_{2} X_{2}}$.

In Corollary 1, condition (4) requires that receiver 1 is more capable in decoding $V$ (and hence $W_{2}$ ) than receiver 2, which is likely to be satisfied in the very strong regime.

We now study the Gaussian Z-IC. Since $S_{1}$ and $S_{2}$ are jointly Gaussian, $S_{1}$ can be expressed as $S_{1}=d S_{2}+S_{1}^{\prime}$ where $d$ is a constant representing the level of correlation, and $S_{1}^{\prime}$ is independent from $S_{2}$ and $S_{1}^{\prime} \sim \mathcal{N}\left(0, Q_{1}^{\prime}\right)$ with $Q_{1}=$ $d^{2} Q_{2}+Q_{1}^{\prime}$. Thus, without loss of generality, the channel model can be expressed in the following equivalent form that is more convenient for analysis,

$$
\begin{aligned}
& Y_{1}=X_{1}+a X_{2}+d S_{2}+S_{1}^{\prime}+N_{1} \\
& Y_{2}=X_{2}+S_{2}+N_{2} .
\end{aligned}
$$

Following Corollary 1, we characterize the channel parameters under which both the states and interference can be fully canceled, and hence the capacity region for the Z-IC is obtained.

Theorem 1. For the state-dependent Gaussian Z-IC with states noncausally known at both transmitters, if the channel parameters $\left(a, d, P_{1}, P_{2}, Q_{1}^{\prime}, Q_{2}\right)$ satisfy the following condition:

$$
\begin{aligned}
& \frac{P_{1}+a^{2} P_{2}+d^{2} Q_{2}+Q_{1}^{\prime}+1}{\left.(d+a \beta)^{2} Q_{2} P_{2}+\left(P_{2}+\beta^{2} Q_{2}\right)\left(P_{1}+Q_{1}^{\prime}+1\right]\right)} \\
& \geqslant \frac{P_{2}+1}{P_{2}}
\end{aligned}
$$

where $\beta=\frac{P_{2}}{P_{2}+1}$, then the capacity region is characterized by (2).

Outline of Proof. Theorem 1 follows from Corollary 1 by setting $U=X_{1}+\alpha_{1} S_{2}+\alpha_{2} S_{1}^{\prime}$, and $V=X_{2}+\beta S_{2}$, where $X_{1}, X_{2}, S_{1}^{\prime}$ and $S_{2}$ are independent Gaussian variables with mean zero and variances $P_{1}, P_{2}, Q_{1}^{\prime}$ and $Q_{2}$, respectively, and $\alpha_{1}, \alpha_{2}$ and $\beta$ satisfy

$$
\alpha_{1}=\frac{P_{1}}{P_{1}+1}\left(d-\frac{a P_{2}}{P_{2}+1}\right), \quad \alpha_{2}=\frac{P_{1}}{P_{1}+1}, \quad \beta=\frac{P_{2}}{P_{2}+1} .
$$

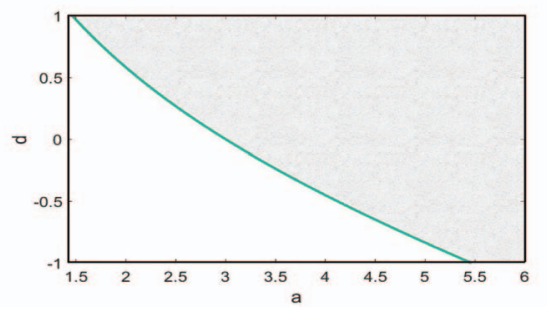

Fig. 2. Characterization of channel parameters $(a, d)$ in shaded area under which the state-dependent Gaussian Z-IC achieves the capacity of the corresponding channel without states and interference in very strong regime.

Based on Theorem 1, if channel parameters satisfy the condition (7), we can simultaneously cancel two states and the interference, and the point-to-point capacity of two receivers without state and interference can be achieved. The correlation between the two states captured by $d$ plays a very important role regarding whether the condition can be satisfied. In Fig. 2, we set $P_{1}=2, P_{2}=2, Q_{1}=1$ and $Q_{2}=1$, and plot the range of the parameter pairs $(a, d)$ under which the channel capacity without states and interference can be achieved. These parameters fall in the shaded area above the line. It can be seen that as $d$ becomes larger (i.e., the correlation between the two states increases), the threshold on the parameter $a$ to fully cancel the interference and state becomes smaller. This suggests that more correlated states are easier to cancel together with the interference.

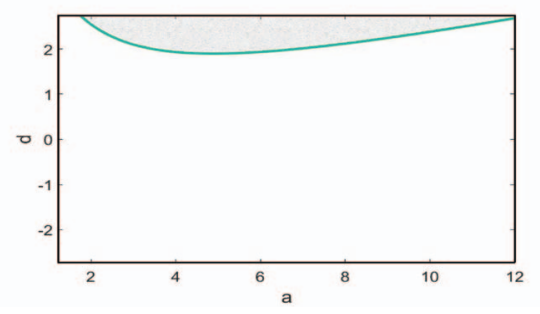

Fig. 3. Characterization of channel parameters $(a, d)$ in shaded area under which the state-dependent Gaussian Z-IC achieves the capacity of the corresponding channel without states in very strong regime when $Q_{2}>\frac{1+P_{2}}{P_{2}}$.

Fig. 2 agrees with the result of the very strong IC without states in the sense that once $a$ is above a certain threshold (i.e., the interference is strong enough), then the point-topoint channel capacity without interference can be achieved. However, this is not always true for the state-dependent Z-IC. This can be seen from the condition (7) in Theorem 1. If we let $a$ go to infinity, then the condition (7) becomes $Q_{2}>\frac{1+P_{2}}{P_{2}}$, which is not always satisfied. This is because in the existence of state, $Y_{1}$ decodes $V$ instead of $X_{2}$, and the decoding rate is largest if the dirty paper coding design of $V$ (based on $S_{2}$ in receiver 2) also happens to be the same dirty paper coding design against $S_{2}$ in receiver 1. Clearly, as $a$ gets too large, $V$ is more deviated from such a favorable design, and hence the decoding rate becomes smaller, which consequently hurts the achievability of the point-to-point capacity for receiver 2 . Such a phenomena can be observed in Fig. 3, where the parameters $(a, d)$ under which the point-to-point channel capacity without interference and states can be achieved fall in the shaded area above the line. It can be seen that the constant $a$ cannot be too large to guarantee the achievability of the point-to-point channel capacity. Furthermore, the figure also suggests that further correlated states allow a larger range of $a$ under which the point-to-point channel capacity can be achieved.

\section{Strong Interference Regime}

For the sake of technical convenience, in this section, we express $S_{2}$ as $S_{2}=c S_{1}+S_{2}^{\prime}$, where $c$ is a constant representing the level of correlation, and $S_{2}^{\prime}$ is independent from $S_{1}$ with 
$S_{2}^{\prime} \sim \mathcal{N}\left(0, Q_{2}^{\prime}\right)$ and $Q_{2}=c^{2} Q_{1}+Q_{2}^{\prime}$. Hence, the channel model can be expressed in the following equivalent form,

$$
\begin{aligned}
& Y_{1}=X_{1}+a X_{2}+S_{1}+N_{1} \\
& Y_{2}=X_{2}+c S_{1}+S_{2}^{\prime}+N_{2} .
\end{aligned}
$$

It has been known that for the corresponding Z-IC without state which is strong but not very strong, i.e., $1 \leqslant a^{2}<1+P_{1}$, the channel capacity contains rate pairs $\left(R_{1}, R_{2}\right)$ satisfying

$$
\begin{aligned}
& R_{1}+R_{2} \leqslant \frac{1}{2} \log \left(1+P_{1}+a^{2} P_{2}\right) \\
& R_{1} \leqslant \frac{1}{2} \log \left(1+P_{1}\right), \quad R_{2} \leqslant \frac{1}{2} \log \left(1+P_{2}\right)
\end{aligned}
$$

which is illustrated as the pentagon O-A-B-E-F in Fig. 4. Our

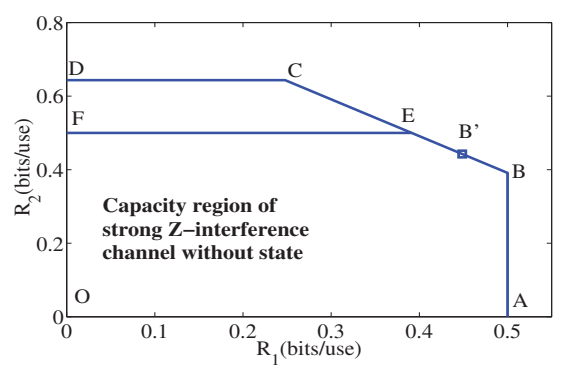

Fig. 4. Capacity region of the strong Z-IC without state

goal here is to study whether the points on the sum-capacity boundary of the Z-IC without state (i.e., the line B-E in Fig. 4) can be achieved for the corresponding state-dependent Z-IC. Such a problem has been studied in [6] for the channel with two receivers corrupted by the same but differently scaled state. Here, we generalize such a study to the situation when the two receivers are corrupted by two correlated states.

Since every point on this line can be achieved by rate splitting and successive cancellation in the case without state, for the state-dependent channel, we continue to adopt the idea of rate splitting and successive cancellation but using auxiliary random variables to incorporate dirty paper coding to further cancel state successively. More specifically, transmitter 1 splits its message $W_{1}$ into $W_{11}$ and $W_{12}$, and then encodes them into $U_{1}$ and $U_{2}$ respectively based on the Gel'fand-Pinsker binning scheme. Then transmitter 2 encodes its message $W_{2}$ into $V$, based on the Gel'fand-Pinsker binning scheme. The auxiliary random variables $U_{1}, U_{2}$, and $V$ are designed such that decoding of them at receiver 1 successively fully cancels the state corruption of $Y_{1}$ so that the sum capacity boundary (i.e., the line B-E) can be achieved if only decoding at receiver 1 is considered. Now further incorporating the decoding at receiver 2, if for any point on the line B-E, decoding of $V$ at receiver 2 does not cause further rate constraints, then such a point is achievable for the state-dependent Z-IC.

Proposition 2. For the state-dependent Z-IC with states noncausally known at both transmitters, if the following condition is satisfied

$$
I\left(V ; U_{1} Y_{1}\right) \leqslant I\left(V ; Y_{2}\right),
$$

then an achievable region consists of rate pairs $\left(R_{1}, R_{2}\right)$ satisfying:

$$
\begin{aligned}
& R_{1} \leqslant I\left(U_{1} ; Y_{1}\right)+I\left(U_{2} ; V Y_{1} \mid U_{1}\right)-I\left(S_{1} ; U_{1} U_{2}\right) \\
& R_{2} \leqslant I\left(V ; U_{1} Y_{1}\right)-I\left(S_{1} ; V\right)
\end{aligned}
$$

for some distribution $P_{S_{1} S_{2}} P_{V \mid S_{1}} P_{X_{2} \mid V S_{1}} P_{U_{1} \mid S_{1}} P_{U_{2} \mid S_{1} U_{1}}$ $P_{X_{1} \mid S_{1} U_{1} U_{2}} P_{Y_{1} \mid S_{1} X_{1} X_{2}} P_{Y_{2} \mid S_{2} X_{2}}$.

We note that although the above achievable rate region does not explicitly contain $S_{2}$, in fact $S_{2}$ implicitly affects the condition (10) via $Y_{2}$. Furthermore, the correlation between $S_{1}$ and $S_{2}$ is also expected to affect the condition (10) via $Y_{2}$, which is our major interest in the Gaussian case.

For the Gaussian model, based on Proposition 2, we characterize the condition under which any point on the sum capacity boundary of the strong Z-IC without states (e.g., point $B^{\prime}$ in Fig. 4) is achievable. Hence, such a point is on the sum capacity boundary of the state-dependent Z-IC.

Theorem 2. For the state-dependent Gaussian Z-IC with states noncausally known at both transmitters, if the channel parameters $\left(a, c, P_{1}, P_{2}, Q_{1}, Q_{2}^{\prime}\right)$ satisfy the following condition:

$$
\begin{aligned}
& \frac{a^{2} P_{2}\left(P_{2}+c^{2} Q_{1}+Q_{2}^{\prime}+1\right)}{(a c-\beta)^{2} Q_{1} P_{2}+\left(a^{2} P_{2}+\beta^{2} Q_{1}\right)\left(Q_{2}^{\prime}+1\right)} \\
& \geqslant 1+\frac{a^{2} P_{2}}{P_{1}^{\prime \prime}+1}
\end{aligned}
$$

where $\beta=\frac{a^{2} P_{2}}{P_{1}+a^{2} P_{2}+1}$, then the following point (on the line $B-E)$

$$
\begin{aligned}
R_{1} & =\frac{1}{2} \log \left(1+\frac{P_{1}^{\prime}}{a^{2} P_{2}+P_{1}^{\prime \prime}+1}\right)+\frac{1}{2} \log \left(1+P_{1}^{\prime \prime}\right) \\
R_{2} & =\frac{1}{2} \log \left(1+\frac{a^{2} P_{2}}{P_{1}^{\prime \prime}+1}\right)
\end{aligned}
$$

where $P_{1}^{\prime}=P_{1}-P_{1}^{\prime \prime}$, is on the sum-capacity boundary.

Outline of Proof. Theorem 2 follows from Proposition 2 by setting $U_{1}=X_{1}^{\prime}+\alpha_{1} S_{1}, U_{2}=X_{1}^{\prime \prime}+\alpha_{2} S_{1}, V=a X_{2}+\beta S_{1}$ where $X_{1}^{\prime}, X_{1}^{\prime \prime}$ and $X_{2}$ are independent Gaussian variables withe mean zero and variances $P_{1}^{\prime}, P_{1}^{\prime \prime}, P_{2}$, respectively, $X_{1}=$ $X_{1}^{\prime}+X_{1}^{\prime \prime}$, and setting $\alpha_{1}, \beta$ and $\alpha_{2}$ respectively for $Y_{1}, Y_{1}^{\prime}$ and $Y_{1}^{\prime \prime}$ to cancel the states via dirty paper coding, where $Y_{1}^{\prime}=Y_{1}-U_{1}=X_{1}^{\prime \prime}+a X_{2}+\left(1-\alpha_{1}\right) S_{1}+N_{1}$ and $Y_{1}^{\prime \prime}=$ $Y_{1}^{\prime}-V=X_{1}^{\prime \prime}+\left(1-\alpha_{1}-\beta\right) S_{1}+N_{1}$. More specifically, we set the coefficients to satisfy the following conditions,

$$
\begin{aligned}
& \alpha_{1}=\frac{P_{1}^{\prime}}{P_{1}+a^{2} P_{2}+1}, \quad \frac{\alpha_{2}}{1-\alpha_{1}-\beta}=\frac{P_{1}^{\prime \prime}}{P_{1}^{\prime \prime}+1}, \\
& \frac{\beta}{1-\alpha_{1}}=\frac{a^{2} P_{2}}{P_{1}^{\prime \prime}+a^{2} P_{2}+1},
\end{aligned}
$$

which can be solved out easily.

Theorem 2 provides the condition of channel parameters under which a certain given point is on the sum-capacity boundary of the capacity region. We next characterize a line 
segment on the sum-capacity boundary for a given set of channel parameters.

Corollary 2. For the state-dependent Z-IC with states noncausally known at both transmitters, if a point on the line $B-E$ in Fig. 4 is on the sum-capacity boundary for a given set of channel parameters, then the segment between this point and point $B$ on the line $B-E$ is on the sum-capacity boundary for the same set of channel parameters.

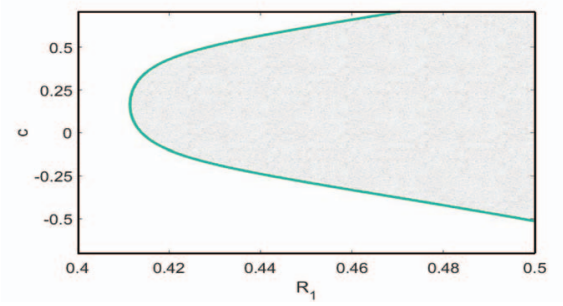

Fig. 5. Ranges of $c$ under which points on sum-capacity boundary of the strong Z-IC without states can be achieved by the state-dependent Z-IC.

In order to numerically illustrate Theorem 2, we first note that each point on the sum-capacity boundary (i.e., the line BE in Fig. 4) can be expressed as $\left(R_{1}, R_{2}\right)=\left(R_{1}, \frac{1}{2} \log \left(P_{1}+\right.\right.$ $\left.\left.a^{2} P_{2}+1\right)-R_{1}\right)$. We now set $P_{1}=1, P_{2}=1, Q_{1}=2, Q_{2}=1$ and $a=1.2$, and hence $R_{1} \in\left[\frac{1}{2} \log (1.72), 0.5\right]$ parameterizes all points from point $E$ to point $B$ in Fig. 4. In Fig. 5, we plot the ranges of $c$ under which points parameterized by $R_{1}$ on the sum capacity boundary of the strong Z-IC without states can be achieved by the state-dependent channel following Theorem 2. It can be seen that as the correlation between the two states (represented by $c$ ) increases, initially more points on the sum-capacity boundary are achieved and then less points are achieved as $c$ is above a certain threshold. Thus, higher correlation does not guarantee more capability of achieving the sum-capacity boundary. This is because in our scheme $U_{1}$, $U_{2}$ and $V$ are specially designed for $Y_{1}$ based on dirty paper coding. At receiver 2, such design of $V$ initially approximates better the dirty paper coding design for $Y_{2}$ as $c$ becomes large, but then becomes worse as $c$ continues to increase, and hence decoding of $V$ at receiver 2 initially gets better and then becomes less capable, which consequently determines variation of achievability of the sum-capacity boundary.

\section{WEAK INTERFERENCE REGIME}

It has been shown in [13] that for the weak Gaussian Z-IC without state, i.e., $a^{2} \leqslant 1$, the sum-capacity can be achieved by treating interference as noise at the interfered receiver. For the state-dependent Z-IC, if the two transmitters independently design dirty paper coding to cancel the state at their corresponding receivers, then the interference-free receiver achieves the capacity of the channel without state, and the interfered receiver (i.e., receiver 1) achieves the same rate as the channel without state by decoding its message treating the interference as noise. Thus, we obtain the following theorem.
Theorem 3. For the state-dependent Z-IC with states noncausally known at both transmitters, if $a^{2} \leqslant 1$, the sumcapacity is given by

$$
C_{\text {sum }}=\frac{1}{2} \log \left(1+\frac{P_{1}}{a^{2} P_{2}+1}\right)+\frac{1}{2} \log \left(1+P_{2}\right) .
$$

It can be seen that the sum-capacity achieving scheme does not depend on the correlation of the states, and hence, in the weak regime, the sum-capacity is not affected by the correlation of the states.

\section{CONCLUSION}

In this paper, we studied the state-dependent Gaussian ZIC with receivers corrupted by two correlated states which are noncausally known at transmitters. We characterized conditions on the channel parameters under which the statedependent Z-IC achieves the capacity region or sum-capacity of the corresponding channel without state. One future work is to generalize our study to the state-dependent regular IC. We also anticipate that the state cancellation schemes we develop here can be useful for studying other state-dependent models.

\section{ACKNOWLEDGMENT}

The work of Y. Sun and Y. Liang was supported by NSF Grant CCF-1618127. The work of S. Shamai was supported by the European Union's Horizon 2020 Research And Innovation Programme with grant agreement no. 694630.

\section{REFERENCES}

[1] L. Zhang, J. Jiang, and S. Cui. Gaussian interference channel with state information. IEEE Trans. Wireless Commun., 12(8):4058-4071, August 2013.

[2] L. Zhang, T. Liu, and S. Cui. Symmetric Gaussian interference channel with state information. In Proc. 49th Annual Allerton Conference on Communication, Control, and Computing, September 2011.

[3] S. Ghasemi-Goojani and H. Behroozi. On the achievable rateregions for state-dependent Gaussian interference channel. Available at http://arxiv.org/abs/1301.5535, submitted in January 2013.

[4] A. Somekh-Baruch, S. Shamai (Shitz), and S. Verdú. Cognitive interference channels with state information. In Proc. IEEE Int. Symp. Information Theory (ISIT), Toronto, Canada, July 2008.

[5] R. Duan and Y. Liang. Bounds and capacity theorems for cognitive interference channels with state. IEEE Trans. Inform. Theory, 61(1):280304, January 2015.

[6] R. Duan, Y. Liang, and S. Shamai (Shitz). State-dependent gaussian interference channels: Can state be fully canceled? IEEE Trans. Inform. Theory, 62:1957 - 1970, April 2016.

[7] R. Duan, Y. Liang, A.Khisti, and S. Shamai (Shitz). State-dependent Gaussian Z-channel with mismatched side-information and interference. In Proc. IEEE Information Theory Workshop (ITW), Sevilla, Spain, September 2013.

[8] S. Ghasemi-Goojani and H. Behroozi. State-dependent Gaussian Zinterference channel: New results. In Proc. IEEE Int. Symp. Information Theory and Its Applications(ISITA), pages 468-472, Victoria, Australia, October 2014.

[9] H. Fehri and H. K. Ghomash. Z-interference channel with side information at the transmitters. AEU - International Journal of Electronics and Communications, 69(9):1167-1180, 2015.

[10] S. Hajizadeh, M. Monemizadeh, and E. Bahmani. State-dependent Z channel. In Proc. Conf. on Information Sciences and Systems (CISS), Princeton, NJ, USA., March 2014.

[11] S. Gel'fand and M. Pinsker. Coding for channels with ramdom parameters. Probl. Contr. Inf. Theory, 9(1):19-31, January 1980.

[12] M. H. M. Costa. Writing on dirty paper. IEEE Trans. Inform. Theory, 29(3):439-441, May 1983.

[13] I. Sason. On achievable rate regions for the Gaussian interference channel. IEEE Trans. Inform. Theory, 50(6):1345-1356, June 2004. 